\title{
Weak covering and the tree property*
}

\author{
Ralf-Dieter Schindler ${ }^{\dagger}$
}

September 12, 2018

\begin{abstract}
Suppose that there is no transitive model of $Z F C+$ there is a strong cardinal, and let $K$ denote the core model. It is shown that if $\delta$ has the tree property then $\delta^{+K}=\delta^{+}$and $\delta$ is weakly compact in $K$.

Let $\neg L^{\text {strong }}$ denote the assumption that there is no transitive (set or proper class sized) model of $Z F C+$ there is a strong cardinal. We have shown in 99 (cf. Theorem 5 ) that $K^{c}$ correctly computes successors of weakly compact cardinals, provided that $\neg L^{\text {stong }}$ holds. Here, $K^{c}$ is the countably complete core model below a strong cardinal. In fact, a straightforward adaptation of the argument given in [9] also yields that $K$, the true core model below a strong cardinal, has the same weak covering property. (Schimmerling had earlier proved this for the core model below a Woodin cardinal, cf. [7]. See [3], 8], or [11] on the theory of $K^{c}$ and $K$.)

The purpose of this note is to prove a more general result, namely the following

THEOREM. If $\neg L^{\text {strong }}$ holds then $\delta^{+K}=\delta^{+}$for every cardinal $\delta$ having the tree property, and such a $\delta$ is weakly compact in $K$.

A cardinal $\delta$ is said to have the tree property if there is no Aronszajn $\delta$-tree, i.e., if every tree of height $\delta$ whose levels all have size less than $\delta$ has a cofinal branch. It is elementary that $\omega$ has the tree property but $\omega_{1}$ does

*1991 Mathematics subject classification: 03E35, 03E45, and 03E55.

$\dagger$ The author gratefully acknowledges a grant of the Deutsche Forschungsgemeinschaft (DFG)
\end{abstract}


not. By [4], $\omega_{2}$ (and in fact for example every double successor) may have the tree property, given the consistency of a weakly compact cardinal.

It is more difficult to show that $\omega_{2}$ and $\omega_{3}$ may simultaneously have the tree property (cf. [1]). In fact, Foreman and Magidor have shown in unpublished work that at least a Woodin cardinal seems to be neccessary to do the job. Our Theorem may be viewed as saying that one reason for the difficulty in forcing $\omega_{2}$ and $\omega_{3}$ to simultaneously have the tree property is that weak covering not only holds for singular and weakly compact cardinals (cf. [3], 9], viz. [5], [7]), but also for cardinals admitting the tree property.

We expect our Theorem to generalize to much higher core models, but we do not at the moment know how to prove it for Steel's core model below one Woodin cardinal, say.

The proof of the Theorem consists in applying the following lemmata to the core model. The first of them is a simple observation building upon a classical insight of Jensen. For its statement, recall Jensen's principle $\square_{\kappa}^{\star}$, cf. [2], p. 283.

LEMMA 1.1. Let $\delta$ have the tree property. Suppose $W$ to be an inner model such that for some $W$-cardinal $\kappa$ we have $W \models 2^{\kappa}=\kappa^{+} \wedge \square_{\kappa}^{\star}$. Then $\delta \neq \kappa^{+W}$. In particular, $\delta$ is inaccessible in any inner model $W$ in which GCH and $\square_{\kappa}^{\star}$ hold for all $\kappa<\delta$.

Proof. By [2] p. 283, inside $W$, using $2^{\kappa}=\kappa^{+}$and $\square_{\kappa}^{\star}$ we can construct a special Aronszajn $\kappa^{+}$-tree. So if $\delta=\kappa^{+W}$ then in $V$ there is an Aronszajn $\delta$-tree. Contradiction!

To state the second, and main, lemma, let us introduce the following terminology. Let $\mathcal{H}, \tilde{\mathcal{H}}$ be two models of the same type. We call an elementary embedding $\sigma: \mathcal{H} \rightarrow \tilde{\mathcal{H}} \kappa$-complete (for a cardinal $\kappa \geq \aleph_{1}$ ) iff for every elementary $\tau: \overline{\mathcal{H}} \rightarrow \tilde{\mathcal{H}}$ with $\operatorname{Card}(\overline{\mathcal{H}})<\kappa$ there is an elementary $\pi: \overline{\mathcal{H}} \rightarrow \mathcal{H}$ such that $\sigma \circ \pi(x)=\tau(x)$ for all $x \in \overline{\mathcal{H}}$ such that $\tau(x) \in \operatorname{ran}(\sigma)$. If $\sigma$ is $\aleph_{1}$-complete then it is also called countably complete.

In this situation, in particular, if $\mathcal{H}=(M ; \in, \ldots)$ is a transitive structure then $\tilde{\mathcal{H}}=(\tilde{M} ; E, \ldots)$ is well-founded and we thus may and will identify $\tilde{\mathcal{H}}$ with its transitive collapse. Note that if we had an infinite decreasing $E$ sequence $\ldots E x_{1} E x_{0}$ then we could choose $\tau: \overline{\mathcal{H}} \rightarrow \tilde{\mathcal{H}}$ with $\left\{x_{n}: n<\omega\right\} \subset$ $\operatorname{ran}(\tau)$ and the countable completeness of $\sigma$ would give some $\pi: \overline{\mathcal{H}} \rightarrow \mathcal{H}$ and $\ldots \in \pi \circ \tau^{-1}\left(x_{1}\right) \in \pi \circ \tau^{-1}\left(x_{0}\right)$. Contradiction! 
LEMMA 1.2. Let $\delta$ have the tree property. Let $W$ be an inner model such that $\delta$ is (strongly) inaccessible in $W, H=\left(H_{\delta^{+}}\right)^{W}$ (= the set of all sets in $W$ being hereditarily $\leq \delta$ in $W$ ) has cardinality $\delta$, and $c f\left(\delta^{+W}\right)=\delta$. Then there is a $\delta$-complete $\sigma: H \rightarrow \tilde{H}$ such that sup $\sigma^{\prime \prime} \delta^{+W}<O n \cap \tilde{H}$.

Proof. To commence, we note that every $X \subset \delta^{+W}$ of cardinality $<\delta$ can be covered by some $Y \in W$ of cardinality $<\delta$. [Let w.l.o.g. $\sup (X)>\delta$. As $c f\left(\delta^{+W}\right)=\delta$, there is $g \in W, g: \delta \rightarrow \sup (X)$ bijective. But $\delta$ is regular, as it has the tree property, so $\theta=\sup \left(g^{-1 "} X\right)<\delta$, and $Y=g " \theta \in W$ is such that $X \subset Y$.]

Now let $F: \delta \rightarrow H$ be bijective. By the previous paragraph and $\delta$ 's being inaccessible in $W$ we may pick $\left(A_{\xi}: \xi<\delta\right)$ such that for all $\bar{\xi}<\xi<\delta$ we have $\delta \in A_{\xi} \in W, A_{\bar{\xi}} \subset A_{\xi}, \operatorname{Card}\left(A_{\bar{\xi}}\right)<\operatorname{Card}\left(A_{\xi}\right)<\delta$, and $F " \xi \subset A_{\xi}$. For every $n<\omega$ let $h_{n}: \omega \times H \rightarrow H, h_{n} \in K$, be some $\Sigma_{n+1}$ Skolem function for $H$ being definable over $H$. For every $X \in \mathcal{P}(H) \cap K$ let us write $h[X]$ for $\bigcup_{n<\omega} h_{n} "(\omega \times X)$ where $X \subset H$, noting that $h[X] \prec H$. Trivially, $H=\bigcup_{\xi<\delta} h\left[A_{\xi}\right]$.

We now let $T$ consist of all $(\xi, \eta)$ such that $\xi<\delta<\sup \left(\delta^{+W} \cap h\left[A_{\xi}\right]\right)<$ $\eta<\delta^{+W}$. Note that by $c f\left(\delta^{+W}\right)=\delta$ for every $\xi<\delta$ there are $\delta$ many $\eta$ 's with $(\xi, \eta) \in T$. We consider $T$ as being ordered by setting $(\xi, \eta) \leq_{T}(\tilde{\xi}, \tilde{\eta})$ iff $\xi \leq \tilde{\xi}$ and there is $\pi: h\left[A_{\xi} \cup\{\eta\}\right] \rightarrow h\left[A_{\tilde{\xi}} \cup\{\tilde{\eta}\}\right]$ induced by the requirements $\pi \uparrow A_{\xi}=i d$ and $\pi(\eta)=\tilde{\eta}$.

Set $[\xi, \eta]=\left\{(\xi, \tilde{\eta}) \in T:(\xi, \eta) \leq_{T}(\xi, \tilde{\eta}) \leq_{T}(\xi, \eta)\right\}$, and let $T^{\star}$ be the set of all such $[\xi, \eta]$ 's. Obviously, $\leq_{T}$ induces a tree ordering $\leq_{T^{\star}}$ on $T^{\star}$. In fact, $\left(T^{\star}, \leq_{T^{\star}}\right)$ can be checked to be a $\delta$-tree. [The $\xi^{\prime}$ 's level of $T^{\star}$ consists of nodes of the form $[\xi, \eta]$ for some $\eta$. Now suppose that this level had cardinality $\delta$, say $\left\{\left[\xi, \eta^{i}\right]: i<\delta\right\}$ with $\left[\xi, \eta^{i}\right] \neq\left[\xi, \eta^{j}\right]$ for $i<j<\delta$ are its nodes. Using $\left(2^{\operatorname{Card}\left(A_{\xi}\right)+\aleph_{0}}\right)^{W}<\delta$ and the pigeonhole principle we may then find $i<j<\delta$ such that $\left(\xi, \eta^{i}\right) \leq_{T}\left(\xi, \eta^{j}\right) \leq_{T}\left(\xi, \eta^{i}\right)$. Contradiction!]

Now let $b$ be any cofinal branch thru $T^{\star}$ given by the tree property of $\delta$. Let us write $\pi_{\xi, \tilde{\xi}}$ for $\pi: h\left[A_{\xi} \cup\{\eta\}\right] \rightarrow h\left[A_{\tilde{\xi}} \cup\{\tilde{\eta}\}\right]$ given by $[\xi, \eta] \leq_{T^{\star}}[\tilde{\xi}, \tilde{\eta}] \in b$. Let $\left(\tilde{H}, \pi_{[\xi, \eta], b}\right)$ be the direct limit of the system $\left(h\left[A_{\xi} \cup\{\eta\}\right], \pi_{\xi, \tilde{\xi}}\right)$. We may define $\sigma: H \rightarrow \tilde{H}$ by sending $x \in H$ to that thread having eventually constant value $x$.

It is now easy to check that $\sigma$ is as desired. Let $\tau: \bar{H} \rightarrow \tilde{H}$ be elementary such that $\bar{H}$ has cardinality $<\delta$. Using the regularity of $\delta, \operatorname{ran}(\tau) \subset$ $\operatorname{ran}\left(\pi_{[\xi, \eta], b}\right)$ for some $[\xi, \eta] \in b$. Then $\pi: \bar{H} \rightarrow H$ is well-defined and elementary where we set $\pi(x)=h_{n}(m, \gamma)$ for $\tau(x)=\pi_{[\xi, \eta], b}\left(h_{n}(m, \gamma)\right), n, m<\omega$, 
$\gamma \in A_{\xi} \cup\{\eta\}$. Moreover, $\tau(x) \in \operatorname{ran}(\sigma)$ means that $\tau(x)=\pi_{[\xi, \eta], b}\left(h_{n}(m, \gamma)\right)$ for some $n, m<\omega$ and some $\gamma \in A_{\xi}$; but then $\sigma \circ \pi(x)=\tau(x)$.

We also have that the thread given by the $\eta$ 's for $[\xi, \eta] \in b, \xi<\delta$, is above every thread having constant value $\zeta$ for any $\zeta<\delta^{+W}$ which implies that sup $\sigma " O n \cap H=\sup \sigma^{\prime \prime} \delta^{+W}<O n \cap \tilde{H}$.

Requiring also that $\delta \cap h\left[A_{\xi}\right]$ is an ordinal, we could have arranged that $\sigma\left\lceil\delta=i d\right.$. Moreover, by replacing the requirement $\xi<\delta<\sup \left(\delta^{+W} \cap\right.$ $\left.h\left[A_{\xi}\right]\right)<\eta<\delta^{+W}$ by $\xi<\delta$ and $\sup \left(\delta \cap h\left[A_{\xi}\right]\right)<\eta<\delta$ we can arrange that in fact $\delta$ is the critical point of $\sigma$ (then $T^{\star}$ resembles the tree constructed in the proof of Lemma 2 in [10]), and we obtain the following

Lemma 1.3. Let $\delta$ have the tree property. Let $H$ be a transitive model of $Z F C-\{$ Powerset $\}$ such that $\delta$ is (strongly) inaccessible in $H, H$ has cardinality $\delta$, and $\operatorname{cf}(\mathrm{On} \cap H)=\delta$. Then there is a $\delta$-complete $\sigma: H \rightarrow \tilde{H}$ with critical point $\delta$.

Before now turning toward the proof of the Theorem let us remark that as a matter of fact if $\neg L^{\text {strong }}$ holds then $c f\left(\kappa^{+K}\right) \geq \operatorname{Card}(\kappa)$ for every $\kappa \geq \aleph_{2}$. Jensen has shown this in [3] (Theorem 7) for $\kappa \geq \aleph_{3}$, but the proof of [0] in fact yields this slight strengthening.

Proof of the Theorem. Let us fix a cardinal $\delta$ having the tree property. Let us assume that $\neg L^{\text {strong }}$ holds (in particular $K$, the true core model below one strong cardinal exists), however $\delta^{+K}<\delta^{+}$. We shall derive a contradiction.

Jensen has shown that $\square_{\kappa}$ holds in $K$ for every $K$-cardinal $\kappa$ (cf. [11], where it is even shown that $K \models \square$, i.e., global square holds in $K$ ). In particular, $\square_{\kappa}^{\star}$ holds everywhere and so by Lemma $1.1 \delta$ is inaccessible in $K$. By the above remark, $c f\left(\delta^{+K}\right) \geq \delta$, and so $c f\left(\delta^{+K}\right)=\delta$. Hence by Lemma 1.2 , setting $H=\left(H_{\delta^{+}}\right)^{K}$, the set of all sets in $K$ being hereditarily $\leq \delta$ in $K$, we may choose a countably complete

$$
\sigma: H \rightarrow \tilde{H} .
$$

Claim. $\tilde{H}$ is a mouse. 
Proof. This is a standard argument. Suppose not, and let $\mathcal{I}$ be a putative iteration of $\tilde{H}$ with a last ill-founded model. Let $\theta$ be large enough and let $\tau^{\star}: \bar{V} \rightarrow V_{\theta}$ be elementary such that $\bar{V}$ is countable and transitive, and $\{\tilde{H}, \mathcal{I}\} \subset \operatorname{ran}\left(\tau^{\star}\right)$. By absoluteness, $\overline{\mathcal{I}}=\tau^{\star-1}(\mathcal{I})$ is a (countable) putative iteration of $\bar{H}=\tau^{\star-1}(\tilde{H})$ with a last ill-founded model. On the other hand, setting $\tau=\tau^{\star} \uparrow \bar{H}$, by the countable completeness of $\sigma$ there is an elementary $\pi: \bar{H} \rightarrow H$ with $\sigma \circ \pi(x)=\tau(x)$ for all $x \in \bar{H}$ such that $\tau(x) \in \operatorname{ran}(\sigma)$, so that $\overline{\mathcal{I}}$ can be copied to give an iteration $\mathcal{I}_{0}$ of $H$. But $H$ is a mouse, so the last model of $\mathcal{I}_{0}$ is well-founded. But then the last model of $\overline{\mathcal{I}}$ is well-founded, too. Contradiction!

(Claim)

We now let $W=U l t(K ; \sigma)$, the ultrapower of $K$ using $\sigma$ as an extender (cf. for example [G] §2.5), and we let

$$
\tilde{\sigma}: K \rightarrow W
$$

denote the associated ultrapower map. We have that $\sigma(\delta)^{+W}=\tilde{\sigma}(\delta)^{+W}=$ sup $\tilde{\sigma} " \delta^{+K}=\sup \sigma^{\prime \prime} \delta^{+K}$. Moreover, $W$ is a universal weasel. [ $K$ correctly computes cofinally many in On successors, and so does $W$. But this implies the universality of $W$ by $\neg L^{\text {strong }}$.]

We may now coiterate $W$ with $\tilde{H}$. As $W$ is universal, there can be no truncation on the $\tilde{H}$-side of the coiteration. As $\neg L^{\text {strong }}, \delta$ is not overlapped in $K$, i.e., there is no $K$-measurable $\mu<\delta$ such that $\mu$ is a strong cardinal in $\mathcal{J}_{\delta}^{K}$. [Otherwise we have found a transitive model of $Z F C+$ there is a strong cardinal.] So $\sigma(\delta)$ is not overlapped neither in $W$ nor in $\tilde{H}$. Hence the coiteration is above $\sigma(\delta)$ on the $\tilde{H}$-side in the sense that the critical points of all extenders used are $\geq \sigma(\delta)$. But $\sigma(\delta)$ is the largest cardinal of $\tilde{H}$, so that in fact $\tilde{H}$ is not moved at all in this coiteration.

Hence we know that $\tilde{H}$ is an initial segment of an iterate $W^{\star}$ of $W$, where the iteration giving $W^{\star}$ is above $\sigma(\delta)$, too. But now, by the property of $\sigma$ given by Lemma 1.2,

$$
\sigma(\delta)^{+W}=\sup \sigma^{\prime \prime} \delta^{+K}<O n \cap \tilde{H} \leq \sigma(\delta)^{+W^{\star}} \leq \sigma(\delta)^{+W} .
$$

Contradiction!

We have thus shown that $\delta^{+K}=\delta^{+}$, and we are left with having to verify that $\delta$ is weakly compact in $K$. For this it suffices to show that in $K$, for all $S \subset \mathcal{P}(\delta)$ with $\operatorname{Card}(S) \leq \delta$ there is a uniform $\delta$-complete filter deciding $S$. 
Let $S \subset \mathcal{J}_{\alpha}^{K}$ with $\alpha<\delta^{+K}=\delta^{+}$, and pick $\beta>\alpha, \beta<\delta^{+}$, such that $\mathcal{J}_{\beta}^{K} \models \operatorname{Card}(\alpha)=\delta, c f(\beta)=\delta$, and $\mathcal{J}_{\beta}^{K} \prec \mathcal{J}_{\delta^{+}}^{K}$. Pick $f \in \mathcal{J}_{\beta}^{K}$ with $f: \delta \rightarrow \mathcal{P}(\delta) \cap \mathcal{J}_{\alpha}^{K}$ bijective. Using Lemma 1.3 we get a $\delta$-complete

$$
\sigma: \mathcal{J}_{\beta}^{K} \rightarrow \tilde{K}
$$

with critical point $\delta$. Define $U=\left\{X \in \mathcal{P}(\delta) \cap \mathcal{J}_{\alpha}^{K}: \delta \in \sigma(X)\right\}$.

For $X \in \mathcal{P}(\delta)$ we have $X \in U$ iff there is $\xi<\delta$ with $X=f(\xi)$ and $\delta \in \sigma(f)(\xi)$. But $f$ may be coded by a subset of $\delta$ in $\mathcal{J}_{\beta}^{K}$, so $f \in \tilde{K}$.

Thus $U \in \tilde{K}$, and $U$ is coded by a subset $A_{U}$ of $\delta$ in $\tilde{K}$. Now the coiteration of $K$ with $\tilde{K}$ is above $\delta$ on both sides by the same argument that gave above that the coiteration of $W$ with $\tilde{H}$ is above $\sigma(\delta)$ on both sides. Moreover, the coiteration is simple on the $K$-side by the universality of $K$. But this implies that $A_{U} \in K$, and so $U \in K$. Finally, the $\delta$-completeness of $\sigma$ implies that $U$ is $\delta$-complete in $K$, and clearly $U$ is uniform.

(Theorem)

\section{References}

[1] Abraham, U., Aronszajn trees on $\aleph_{2}$ and $\aleph_{3}$, Ann. Pure Appl. Logic 24 (1983), pp. 213 - 230.

[2] Jensen, R. B., The fine structure of the constructible hierarchy, Ann. Math. Logic 4 (1972), pp. 229 - 308.

[3] — , The core model for non-overlapping extender sequences, handwritten notes.

[4] Mitchell, W. J., Aronszajn trees and the independence of the transfer property, Ann. Pure Appl. Logic 5 (1972), pp. 21 - 46.

[5] —, and Schimmerling, E., Covering without countable closure, Math. Research Letters 2 (1995), pp. 595 - 609.

[6] — , and Steel, J., The covering lemma up to a Woodin cardinal, Ann. Pure Appl. Logic.

[7] Schimmerling, E., and Steel, J., The maximality of the core model, preprint. 
[8] Schindler, R.-D. The core model up to one strong cardinal, Ph. D. Thesis, Bonn, 1996.

[9] - Weak covering at large cardinals, Math. Logic Quarterly 43 (1997), pp. $22-28$.

[10] - Successive weakly compact or singular cardinals, Journal Symb. Logic, to appear.

[11] Zeman, Martin, The core model for non-overlapping extender sequences and its applications, Ph. D. Thesis, Berlin, 1997.

Mathematisches Institut

UNI BONN

BERINGSTR. 4

53115 Bonn, GERMAnY

rds@rhein.iam.uni-bonn.de

MATH DEPT.

$\mathrm{UCB}$

BERKELEY, CA 94720, USA

rds@math . berkeley . edu 